\title{
Aproximación a los estilos y estrategias de enseñanza del profesorado de la ESO y Bachillerato de ciencias experimentales y tecnología: un estudio de caso
}

\author{
Carmen Ropero-Padilla ${ }^{1}$ \\ Miguel Rodriguez-Arrastia ${ }^{1}$ (D) \\ Aida Sanahuja Ribés ${ }^{1}$ (D) \\ ${ }^{1}$ Universidad Jaume I (UJI), España
}

Resumen. Los conceptos, los estilos y las estrategias de enseñanza se encuentran íntimamente ligados, quedando supeditados a la experiencia del propio docente. De esta forma, no sólo quedará configurado el aprendizaje del alumnado a partir de las metas que este asuma, sino que estos estilos docentes también tendrán una impronta en diferentes elementos de dicho proceso, como la motivación e implicación en el aprendizaje. El objetivo de este estudio, por tanto, fue conocer los diferentes estilos y su evolución dentro del profesorado de ciencias experimentales y tecnología, así como su impacto en las estrategias de aula. Para ello, se diseñó un estudio cualitativo descriptivo de corte fenomenológico, a partir de un estudio de caso. Se realizaron un total de 6 entrevistas a profesores/as de diferentes niveles educativos dentro del área de ciencias experimentales y tecnología, acompañadas de observaciones no participantes en el aula y análisis documental. Los resultados mostraron datos interesantes sobre el uso de diferentes estilos de enseñanza, más cercanos a un estilo mixto a estrategias de aprendizaje cooperativas. Como conclusión, merece especial atención la evolución de los participantes hacia estilos y estrategias más activas, aunque queda pendiente la tarea de profundizar en el proceso de evaluación y reflexión docente. Palabras clave: ciencias experimentales; entrevistas; estilos de enseñanza; estrategias de enseñanza; profesorado.

Uma aproximação aos estilos e estratégias de ensino dos professores da Educação Secundária Obrigatória (ESO) e do Ensino Médio (Bachillerato) em ciências experimentais e tecnologia: um estudo de caso

Resumo. Os conceitos, estilos e estratégias de ensino estão intimamente ligados, estando sujeitos à própria experiência do professor. Desta forma, não só a aprendizagem dos alunos será configurada pelos objetivos que eles assumem, mas também os estilos de ensino terão um impacto em diferentes elementos deste processo, como a motivação e o envolvimento na aprendizagem. Portanto, o objetivo deste estudo era conhecer os diferentes estilos e a sua evolução nos professores de Ciências Experimentais e Tecnologia, bem como o seu impacto nas estratégias de sala de aula. Para este fim, foi elaborado um estudo qualitativo, descritivo e fenomenológico, com base num estudo de caso. No total, foram realizadas 6 entrevistas com professores de diferentes níveis educacionais na área de Ciências Experimentais e Tecnologia, acompanhadas de observações em sala de aula de não participantes e análise documental. Os resultados mostraram dados interessantes sobre o uso de diferentes estilos de ensino, mais próximos de um estilo misto do que de estratégias de aprendizagem cooperativa. Em conclusão, merece atenção especial a evolução dos participantes em direção a estilos e estratégias mais ativas, embora a tarefa de aprofundar o processo de avaliação e reflexão dos professores ainda esteja pendente.

Palavras-chave: ciências experimentais; entrevistas; estilos de ensino; estratégias de ensino; professores

An approach to teaching styles and strategies for experimental sciences in CSE and Bachelor: a case study

Abstract. Concepts, styles, and teaching strategies are strongly intertwined and are influenced by the experience of the teacher. In this manner, not only will student learning be configured on the basis of the goals that they assume, but these teaching styles will also have an impact on different elements of this process, such as motivation and engagement in learning. Thus, the aim of the study was to explore different teaching styles and their evolution in the area of experimental sciences and technology, as well as their impact on teaching strategies used within the classroom. To this end, a qualitative descriptive study with a phenomenological approach was designed, based on a case study. A total of 6 interviews were carried out with teachers of different educational levels within the area of experimental sciences and technology, as well as non-participant observations in the classroom and documental analysis. The results showed interesting data about the use of different teaching styles, closer to a mixed style and a cooperative learning strategy. In conclusion, the change towards more active styles and strategies deserves special attention, although the challenge of deepening the teacher assessment and reflection process remains. Keywords: experimental sciences; interviews; teachers; teaching strategies; teaching styles 


\section{Introducción}

Hoy día, la enseñanza en ciencias no queda relegada exclusivamente a llevar las propuestas curriculares al aula, sino que implica también una necesidad de conectar con el alumnado. Un alumnado que cada vez es más diverso y donde se requiere que el profesorado actúe como mediador, entre otros aspectos, para promover aprendizajes significativos y el desarrollo de habilidades, actitudes y valores a través de sus estilos y estrategias de enseñanza (García-Cué et al., 2012; González-Peiteado, 2013).

\subsection{De una enseñanza tradicional o académica a una enseñanza moderna o activa}

Diferenciar explícitamente entre enseñanzas tradicionales o académicas y enseñanzas activas o modernas no parece ser del todo correcto, dado que las llamadas "enseñanzas modernas" no son algo realmente novedoso, contando con más de un siglo de antigüedad (Brailovsky, 2018). A pesar de ello, este es un concepto que parece encontrarse aún bastante arraigado entre los docentes y, por ello, conviene diferenciar y definir ambos conceptos. Por un lado, los procesos de enseñanzaaprendizaje con una estrategia mayoritariamente expositiva, desarrollados a través de procesos repetitivos de transmisión de conocimientos, constituyen los modelos pedagógicos denominados como tradicionales, limitando en gran medida los procesos de resolución de problemas y la investigación por parte del alumnado, así como cualquier proceso de creatividad (Parra-Pineda y Bustamante-Ramírez, 2003). De esta manera, en la enseñanza académica o pasiva, el profesorado transmite la información para una lección específica mientras el alumnado la recibe de forma pasiva, sentado, escuchando, copiando, memorizando y repitiendo lo que el docente dice o ha dicho. En definitiva, la trasmisión de conocimiento queda enfocada desde el profesorado al estudiantado (Ivic, 2016)".

Por el contrario, la enseñanza activa o centrada en el alumnado, implica que los profesores trabajen junto al alumnado para seleccionar los objetivos de enseñanza y las tareas basadas en problemas reales, así como en los conocimientos previos del estudiante, sus experiencias e intereses. Este tipo de enseñanza está enfocada al desarrollo de habilidades y competencias, fomentando estrategias cognitivas de un nivel elevado como la resolución de problemas, así como recoger, clarificar, interpretar y transmitir información. En este contexto, el alumnado toma la responsabilidad de su aprendizaje, buscando conocimiento de manera activa, construyéndolo y dándole significado. Esta forma de enseñar, por ende, crea las condiciones necesarias para preparar al alumnado para una vida activa dentro de la sociedad en la que se relaciona (Alonso et al., 2012; Ivic, 2016), al mismo tiempo que se fomenta la denominada competencia científica en el alumnado, esto es, aprender a conocer, hacer, hablar o saber de ciencia (Cañal de León, 2012; Cañas et al., 2014; García-Barros et al., 2021; Verde-Romera et al., 2017).

Al hilo de lo planteado, si bien es verdad que en la práctica el concepto de enseñanza y aprendizaje no se suelen separar, parece oportuno centrarse en definir concretamente qué se entiende por estrategias de enseñanza, dada su importancia en el propio proceso de aprendizaje del alumnado. De esta manera, Montes de OcaRecio y Machado-Ramírez (2011) definen las estrategias de enseñanza como los 
procedimientos utilizados por el docente para promover aprendizajes significativos. A razón de esta definición, las estrategias de enseñanza deben guiar las acciones del estudiante para conseguir las diferentes metas de aprendizaje, además de ser flexibles y adaptativas a las distintas circunstancias de enseñanza, en vez de acciones automáticas y rígidas (Parra-Pineda y Bustamante-Ramírez, 2003).

En este sentido, dentro de las taxonomías de estrategias de enseñanza- aprendizaje, se encuentran aquellas centradas en el alumno, las centradas en el docente, las centradas en el proceso y las centradas en el objeto de conocimiento. Entre todas ellas, las estrategias centradas en el alumnado parecen señalarse como las más efectivas para un aprendizaje significativo, como pueden ser el método de problemas, de casos, de indagación, de proyectos, educación STEM o juegos de roles, entre otros, fundamentándose en el autoaprendizaje, así como en el desarrollo del pensamiento y el razonamiento crítico (Aguilera et al., 2021; Azorín-Abellán, 2018). Asimismo, las metodologías participativas parecen incidir de manera favorable en aspectos cognitivos (rendimiento académico), afectivos (actitud hacia la ciencia) y procedimentales (método científico) de los estudiantes (Aguilera-Morales y Perales-Palacios, 2016). No obstante, y en relación con la aplicación de esta enseñanza, cabe destacar el informe TALIS, donde se señalan diversos aspectos que pueden influir y dificultar la calidad de esta. Entre ellos, se subrayan la escasez de personal de apoyo, la dificultad de poder alcanzar una equidad en alumnado con necesidades educativas especiales por falta de profesorado e inadecuación de espacios y materiales, así como una falta de capacitación para la enseñanza en entornos multiculturales y plurilingües (Ministerio de Educación y Formación Profesional, 2019). Como apuntan González-Peiteado y Pino-Juste (2016), tampoco se puede obviar la existencia de diferencias individuales dentro de los estilos de enseñanza, como son los valores, ideas, técnicas, métodos y experiencias propias que terminan moldeando el estilo de enseñanza particular de cada docente. Todo ello hace que el docente se relacione con los elementos didácticos de una determinada manera, influyendo irremediablemente y de manera significativa en el aprendizaje del alumnado, así como en la calidad del proceso. En este sentido, existen diferentes perspectivas para analizar el pensamiento y las conductas del profesorado, su evolución y el impacto en el aprendizaje. En relación con el pensamiento, relacionado con las creencias que tiene el profesorado, se puede analizar a través de los "modelos didácticos", mientras que el análisis relacionado con las conductas, es decir, relacionado con las estrategias llevadas a cabo por el profesorado, se puede describir mediante los "estilos de enseñanza" (Contreras-Palma, 2009; Martínez-Aznar et al., 2002).

\subsection{Delimitación conceptual: estrategias y estilos docentes}

Como plantean García-Cué et al. (2012) en su trabajo sobre la mejora en el proceso de enseñanza-aprendizaje, las estrategias y los estilos docentes se encuentran íntimamente relacionados, quedando en gran parte supeditadas las estrategias de enseñanza a cómo el docente considera que el alumnado puede adquirir, almacenar y utilizar el conocimiento. En este contexto, la clasificación elaborada por DelgadoNoguera (1996) cobra especial interés, sugiriendo una gradación en la cercanía a un estilo global activo, según se incorporen más o menos dimensiones o componentes del enfoque. Tal y como queda reflejado en su trabajo, este marco teórico permite 
diferenciar entre un estilo académico y un estilo activo, integrando en este último otros estilos como el reflexivo, individualizador, cooperador, indagador e innovador (Tabla 1). Así, se considera que cada docente puede tener un estilo predominante, pudiendo además combinarse con otros estilos para poder adaptarse a las particularidades de los estudiantes al no ser estilos excluyentes entre sí (Delgado-Noguera, 1991).

Tabla 1. Estilos de enseñanza y sus características

\begin{tabular}{|c|c|}
\hline Estilos & Características \\
\hline \multirow{5}{*}{$\begin{array}{l}\text { Estilo académico o } \\
\text { tradicional }\end{array}$} & Se ampara en el orden, la tarea y el control exhaustivo \\
\hline & El docente toma las decisiones \\
\hline & No se potencia la participación activa \\
\hline & No existe individualización, sino respuesta colectiva \\
\hline & No promueven relaciones afectivas \\
\hline \multirow[t]{4}{*}{ Estilo reflexivo } & Se promueve una mayor autonomía en el alumnado \\
\hline & Los alumnos intervienen en la toma de decisiones \\
\hline & $\begin{array}{l}\text { Se favorece la implicación de los alumnos en el proceso de aprendizaje: } \\
\text { aprender a pensar }\end{array}$ \\
\hline & $\begin{array}{l}\text { Se confiere más libertad al alumno con la intención de facilitar mayor } \\
\text { capacidad analítica, independencia de criterio y espontaneidad }\end{array}$ \\
\hline \multirow[t]{4}{*}{ Estilo indagador } & Docente como guía \\
\hline & Se promueve la actitud de curiosidad \\
\hline & $\begin{array}{l}\text { Se favorecen el aprendizaje por descubrimiento, la capacidad crítica y } \\
\text { la toma de decisiones }\end{array}$ \\
\hline & Se traslada a situaciones reales el proceso de resolución de problemas \\
\hline \multirow[t]{2}{*}{ Estilo cooperador } & $\begin{array}{l}\text { Se promueven la cooperación y la solidaridad a través de la práctica del } \\
\text { trabajo grupal }\end{array}$ \\
\hline & Se fomentan la responsabilidad y un clima de cordialidad \\
\hline \multirow[t]{5}{*}{$\begin{array}{l}\text { Estilo individualiza- } \\
\text { dor }\end{array}$} & $\begin{array}{l}\text { Se persigue atender individualmente al alumnado y proporcionar una en- } \\
\text { señanza que tenga en cuenta sus intereses, motivaciones y capacidades }\end{array}$ \\
\hline & $\begin{array}{l}\text { La enseñanza se adapta al periodo evolutivo y al grado de madurez } \\
\text { adquirida del estudiante }\end{array}$ \\
\hline & $\begin{array}{l}\text { La organización del espacio y el tiempo permite actividades grupales e } \\
\text { individuales }\end{array}$ \\
\hline & Existe flexibilidad en los programas educativos \\
\hline & El docente hace uso de la retroalimentación de manera inmediata \\
\hline \multirow[t]{2}{*}{ Estilo innovador } & Se promueve conseguir un aprendizaje activo y espontáneo \\
\hline & Se respeta el empleo de respuestas inusitadas e ideas originales \\
\hline
\end{tabular}
Fuente: extraído de Delgado-Noguera (1991).

En esta línea, estudios como el de Domingo-Roget (2020) o González-Peiteado y Pino-Juste (2016) empiezan a dibujar en sus resultados la importancia del estilo docente utilizado. De este modo, se señalan los estilos activos como los preferidos por sus participantes, más concretamente los estilos innovador, cooperativo y reflexivo. Todos ellos se relacionan con la espontaneidad, trabajo en grupo, capacidad productiva y situaciones de resolución de problemas, análisis y toma de decisiones. 
De la misma manera, autores como Monarca y Rappoport (2017) profundizan en los tipos de metas que asume el alumnado, así como en su relación con la implicación y motivación de éste en el aprendizaje. De esta manera, estos factores se verán modificados, dependiendo de si el alumnado adopta una meta de rendimiento o de aprendizaje (Navas et al., 2016). No obstante, gran parte de la literatura (García-Fraile y Rojas-Aguilera, 2018; Morales y Camacho-Verdugo, 2020) se ha centrado en el análisis de las estrategias de enseñanza desde la figura del alumnado, observando una escasez en la misma sobre las experiencias y percepciones de los propios docentes en los niveles de secundaria y bachillerato. Por ende, según apuntan Jiménez-Tenorio y Oliva-Martínez (2016) resulta importante disponer de tareas formativas encaminadas a abordar las estrategias didácticas en las ciencias experimentales y tecnología para poder implementar propuestas didácticas innovadoras. Con todo lo comentado hasta el momento, parece importante conocer cuáles son los estilos de enseñanza predominantes a través de la autopercepción de los propios docentes y, sobre todo, explorar cómo han ido adaptándose a lo largo de su carrera profesional para intentar conseguir una enseñanza de calidad.

\section{Objetivos}

El propósito de este estudio, por tanto, fue conocer la caracterización global de distintas dimensiones de pensamiento o acción docente entre el profesorado de Ciencias Experimentales y Tecnología (Biología y Geología, Física y Química, Tecnología e Informática) (CCEE) dentro de la Educación Secundaria Obligatoria (ESO) y Bachillerato de un Instituto de Educación Secundaria (IES) de Castellón, a través de las experiencias y perspectivas de los docentes, y cómo estos estilos influyen en las estrategias utilizadas en el aula.

\section{Metodología}

\subsection{Diseño}

Con el fin de alcanzar el objetivo planteado, se realizó un estudio cualitativo descriptivo de corte fenomenológico, utilizando como referencia el marco teórico de Delgado-Noguera (1996). El estudio se basó en el análisis de contenido de las experiencias y perspectivas de los docentes de CCEE y Tecnología, en el uso de estrategias y estilos de enseñanza utilizadas a lo largo de su carrera profesional como docentes (Creswell y Creswell, 2018). Cabe señalar que el objetivo principal de la investigación cualitativa es entender de manera más amplia un determinado fenómeno educativo (Jorrín-Abellán et al., 2021; Sandín-Esteban, 2003). En este caso y dentro de una investigación cualitativa (Fernández-Navas y Postigo-Fuentes, 2020), se optó por un estudio de caso, con la intención de poder comprender la realidad particular de una experiencia y un contexto determinado (Álvarez-Álvarez y San Fabián-Maroto, 2012). Más aún, cabe señalar que el estudio de caso que se presenta en este trabajo se trata de un estudio intrínseco, ya que éste viene dado e interesa comprender particularmente este fenómeno (Simons, 2009; Stake, 1998). 


\subsection{Participantes y contexto}

El estudio se llevó a cabo en un instituto público en la provincia de Castellón de la Plana (España), entre los meses de enero y marzo de 2020. Este instituto ofrece 3 niveles educativos diferentes: Educación Secundaria Obligatoria, Bachillerato en las modalidades de Humanidades y Ciencias Sociales, Artes y Ciencias y Tecnología, así como Formación Profesional Básica de Electricidad y Electrónica. Concretamente, el estudio se realizó con el profesorado que impartía docencia en los diferentes niveles de ESO y Bachillerato con un itinerario de CCEE y Tecnología. En relación con las asignaturas incluidas dentro de este itinerario, se encontrarían Biología y Geología; Física y Química; Anatomía Aplicada; Informática y Tecnología (Generalitat Valenciana, 2020).

El tipo de muestreo fue intencional, criterial y por conveniencia. Los criterios de inclusión que se establecieron englobaban que (i) fuesen profesorado de los niveles educativos de ESO o Bachillerato, (ii) que estuviesen impartiendo docencia en el momento del estudio en el itinerario de CCEE y Tecnología y (iii) accediesen a participar en el estudio. Finalmente participaron 6 profesores/as de diferentes niveles educativos, con docencia desde primero de ESO hasta segundo de Bachillerato, divididos de forma equitativa entre hombres $(n=3,50 \%)$ y mujeres $(n=3,50 \%)$. El rango de edad de los participantes se encontraba entre 38 y 59 años $(52 \pm 9,07)$. En cuanto a la experiencia laboral de los mismos, la media se encontraba en $24,33 \pm 11,84$ años. Los detalles sociodemográficos quedarían resumidos en la Tabla 2.

Tabla 2. Características sociodemográficas

\begin{tabular}{|c|c|c|c|c|c|c|}
\hline & Edad & Sexo & $\begin{array}{c}\text { Experiencia } \\
\text { laboral (años) }\end{array}$ & $\begin{array}{l}\text { Especialidad } \\
\text { en CCEE y } \\
\text { Tecnología }\end{array}$ & $\begin{array}{l}\text { Situación } \\
\text { laboral }\end{array}$ & $\begin{array}{c}\text { Nivel de } \\
\text { estudios } \\
\text { alcanzado }\end{array}$ \\
\hline P01 & 43 & Mujer & 8 & Física y Química & Sustitución & Licenciatura \\
\hline P02 & 38 & Mujer & 12 & Informática & Fija & Licenciatura \\
\hline P03 & 59 & Hombre & 36 & $\begin{array}{l}\text { Biología y Geo- } \\
\text { logía }\end{array}$ & Fijo & Máster \\
\hline P04 & 58 & Mujer & 30 & $\begin{array}{l}\text { Biología y Geo- } \\
\text { logía }\end{array}$ & Fija & Licenciatura \\
\hline P05 & 57 & Hombre & 25 & $\begin{array}{l}\text { Biología y Geo- } \\
\text { logía }\end{array}$ & Fijo & Licenciatura \\
\hline P06 & 57 & Hombre & 35 & $\begin{array}{l}\text { Biología y Geo- } \\
\text { logía }\end{array}$ & Fijo & Doctorado \\
\hline Media $\pm S D$ & $52 \pm 9,07$ & & $24,33 \pm 11,84$ & & & \\
\hline
\end{tabular}

SD: Desviación típica

La observación no participante tuvo lugar en dos aulas con docentes diferentes que impartían la asignatura de Biología y Geología. Los docentes seleccionados fueron el P04 y el P06. En este sentido, una de las aulas correspondía al curso académico de cuarto de ESO, con un total de 25 alumnos/as, y la otra aula era de segundo de ESO con 24 alumnos/as. 


\subsection{Instrumento y procedimiento de recogida de datos}

Siguiendo el esquema para estudio de casos en la investigación educativa de Martínez-Bonafé (1988), las fases que se llevaron a cabo durante el procedimiento de la investigación quedarían detalladas de la siguiente manera:

- Fase preactiva: en esta fase se planteó el marco teórico que conduce esta investigación y se establecieron los objetivos a abordar. Por otro lado, el centro y los participantes fueron elegidos por cumplir los criterios de inclusión mencionados anteriormente. En este periodo, se solicitó el consentimiento del centro, el consentimiento informado para los docentes, se confeccionaron los instrumentos de recogida de datos, así como el proyecto de investigación para la Comisión Deontológica de la Universidad Jaume I.

- Fase interactiva: durante esta fase, se realizó la recogida de datos una vez que los docentes accedieron a participar de manera voluntaria e hicieron entrega del consentimiento informado, junto a un formulario de datos sociodemográficos. Para la recogida de datos, se llevó a cabo una entrevista con cada docente en el día y hora pactados con cada uno/a según su disponibilidad (Anexo). Estos datos se fueron analizando durante la recogida de datos hasta que se alcanzó la saturación de estos. Asimismo, se recolectaron diferentes documentos propios de sus prácticas docentes con el objetivo de realizar un análisis documental de los mismos, focalizando la observación en 2 aulas diferentes con docentes que impartían la asignatura de Biología y Geología.

- Fase postactiva: en esta última fase se elaboró la transcripción literal de las entrevistas realizadas, asignando un sistema de códigos para la identificación de los datos. Tras ello, el análisis de los datos se confeccionó mediante el software ATLAS.ti (explicado más ampliamente en el apartado Análisis de datos). Terminado este análisis, se elaboró la redacción de los resultados y se consultaron con los diferentes docentes para que tuvieran la oportunidad de proponer, detallar o rectificar aquello que considerasen oportuno.

Como se ha venido comentando, los datos se recogieron haciendo uso de una entrevista semiestructurada elaborada a partir de la literatura revisada (Kallio et al., 2016), permitiendo la manifestación abierta de los entrevistados. La entrevista en la investigación cualitativa trata de obtener información mediante una conversación, con la intención de elaborar un estudio analítico de la investigación (Ruiz-Olabuénaga, 2012). En este estudio, las entrevistas se realizaron de manera individual, llevando a cabo preguntas relacionadas con la práctica docente. Cabe destacar entre ellas, la formación académica y continua, años de experiencia laboral como docente, situación laboral, experiencia en el uso de estrategias de enseñanza y gestión del aula, uso de recursos materiales y humanos, así como el tipo de evaluación y el uso de la autoevaluación en la práctica docente, entre otras. 
Tabla 3. Datos recogidos en la investigación

\begin{tabular}{|c|c|c|c|}
\hline Instrumento & Fuente & Contenido & Soporte \\
\hline \multirow[t]{6}{*}{ Entrevista } & Docente 1 & Entrevista & Grabación audio: 34:58 minutos \\
\hline & Docente 2 & Entrevista & Grabación audio: $38: 50$ minutos \\
\hline & Docente 3 & Entrevista & Grabación audio: $32: 55$ minutos \\
\hline & Docente 4 & Entrevista & Grabación audio: $35: 25$ minutos \\
\hline & Docente 5 & Entrevista & Grabación audio: $40: 52$ minutos \\
\hline & Docente 6 & Entrevista & Grabación audio: $37: 14$ minutos \\
\hline \multirow[t]{3}{*}{$\begin{array}{l}\text { Observación no } \\
\text { participante }\end{array}$} & \multirow[t]{3}{*}{$\begin{array}{l}\text { Situación del } \\
\text { aula }\end{array}$} & \multirow[t]{2}{*}{ Laboratorio } & $\begin{array}{l}\text { Sesión } n^{0} 3 \text { : diario de campo } \\
\text { (Docente 4) }\end{array}$ \\
\hline & & & $\begin{array}{l}\text { Sesión } n^{\circ} 5 \text { : diario de campo } \\
\text { (Docente 6) }\end{array}$ \\
\hline & & $\begin{array}{l}\text { Estrategias de } \\
\text { aprendizaje coope- } \\
\text { rativo }\end{array}$ & $\begin{array}{l}\text { Sesión } n^{0} 7 \text { : diario de campo } \\
\text { (Docente 4) }\end{array}$ \\
\hline \multirow[t]{4}{*}{$\begin{array}{l}\text { Observación no } \\
\text { participante }\end{array}$} & \multirow[t]{4}{*}{$\begin{array}{l}\text { Situación del } \\
\text { aula }\end{array}$} & \multirow{3}{*}{$\begin{array}{l}\text { Estrategias de } \\
\text { aprendizaje coope- } \\
\text { rativo }\end{array}$} & $\begin{array}{l}\text { Sesión } n^{0} 2 \text { : diario de campo } \\
\text { (Docente 6) }\end{array}$ \\
\hline & & & $\begin{array}{l}\text { Sesión } n^{\circ} 8 \text { : diario de campo } \\
\text { (Docente 4) }\end{array}$ \\
\hline & & & $\begin{array}{l}\text { Sesión } n^{0} 10 \text { : diario de campo } \\
\text { (Docente 6) }\end{array}$ \\
\hline & & Debates & $\begin{array}{l}\text { Sesión } n^{\circ} 12 \text { : diario de campo } \\
\text { (Docente 4) }\end{array}$ \\
\hline \multirow[t]{9}{*}{$\begin{array}{l}\text { Análisis docu- } \\
\text { mental }\end{array}$} & \multirow[t]{9}{*}{$\begin{array}{l}\text { Documentos o } \\
\text { materiales }\end{array}$} & $\begin{array}{l}\text { Fichas actividades } \\
\text { grupales }\end{array}$ & $\begin{array}{l}5 \text { fichas de actividades } \\
\text { (Docente 4) }\end{array}$ \\
\hline & & $\begin{array}{l}\text { Murales "Las capas } \\
\text { de la tierra" }\end{array}$ & $\begin{array}{l}1 \text { mural por grupo realizado por el } \\
\text { alumnado } \\
\text { (Docente 6) }\end{array}$ \\
\hline & & $\begin{array}{l}\text { Carteles "Animales } \\
\text { vertebrados e inver- } \\
\text { tebrados" }\end{array}$ & $\begin{array}{l}1 \text { cartel por grupo realizado por el } \\
\text { alumnado } \\
\text { (Docente 6) }\end{array}$ \\
\hline & & $\begin{array}{l}\text { Actividad de gami- } \\
\text { ficación "El cuerpo } \\
\text { humano" }\end{array}$ & $\begin{array}{l}\text { Juego de mesa y reglas del juego } \\
\text { elaborado por el alumnado } \\
\text { (Docente 4) }\end{array}$ \\
\hline & & $\begin{array}{l}\text { Rúbrica evaluación } \\
\text { trabajos escritos }\end{array}$ & $\begin{array}{l}\text { Copia en formato digital } \\
\text { (Docentes } 4 \text { y } 6 \text { ) }\end{array}$ \\
\hline & & $\begin{array}{l}\text { Rúbrica evaluación } \\
\text { exposición oral }\end{array}$ & $\begin{array}{l}\text { Copia en formato digital } \\
\text { (Docentes } 4 \text { y } 6 \text { ) }\end{array}$ \\
\hline & & $\begin{array}{l}\text { Rúbrica evaluación } \\
\text { de laboratorios }\end{array}$ & $\begin{array}{l}\text { Copia en formato digital } \\
\text { (Docentes } 4 \text { y } 6 \text { ) }\end{array}$ \\
\hline & & $\begin{array}{l}\text { Rúbrica de evalua- } \\
\text { ción funcionamiento } \\
\text { y comportamiento } \\
\text { del grupo }\end{array}$ & $\begin{array}{l}\text { Copia en formato digital } \\
\text { (Docentes } 4 \text { y } 6 \text { ) }\end{array}$ \\
\hline & & $\begin{array}{l}\text { Rúbrica evaluación } \\
\text { de libretas }\end{array}$ & $\begin{array}{l}\text { Copia en formato digital } \\
\text { (Docentes } 4 \text { y } 6 \text { ) }\end{array}$ \\
\hline
\end{tabular}


En cuanto a la observación científica, cabe destacar que se contempló como un proceso de atención meticuloso y metódico del fenómeno de estudio (Ruiz-Olabuénaga, 2012). La observación realizada fue no participante (Fuertes-Camacho, 2011). Para el seguimiento de las observaciones, se hizo uso de un diario de campo donde se llevaron a cabo anotaciones descriptivas y objetivas sobre el contexto del aula, la relación entre profesorado y alumnado o las diferentes estrategias de enseñanza desarrolladas, entre otros elementos (Rekalde et al., 2014). Por último, en relación con el análisis documental, éste se llevó a cabo con el objetivo de complementar las entrevistas de los docentes, previamente mencionadas, aportando evidencias documentales (Martínez-Bonafé, 1988). A continuación, en la Tabla 3, se muestran todos los datos recogidos en esta investigación.

\subsection{Análisis de datos}

Todas las anotaciones y grabaciones fueron transcritas e incorporadas a unidades hermenéuticas de análisis, para ser analizadas con la ayuda del programa informático ATLAS.Ti (v9.0). Posteriormente, se realizó un análisis de contenido deductivo siguiendo los pasos de Colaizzi (Rapley, 2014). En primer lugar (i) se realizó un proceso de familiarización a través de la lectura de todas las transcripciones, (ii) identificando las citas significativas en cada entrevista. A continuación, (iii) se realizó la condensación de los enunciados en unidades de significado, antes de (iv) agruparlas en temas comunes. Asimismo, se realizó (v) una descripción del fenómeno, incorporando los temas elaborados en el paso anterior, a través de una (vi) estructura fundamental que condensase los aspectos claves del fenómeno de estudio. Por último, (vii) la estructura fue revisada por los propios participantes, modificando o confirmando la estructura realizada.

Como criterios de elaboración metodológicos, se siguieron los criterios COREQ para investigación cualitativa (Tong et al., 2007). Del mismo modo, se siguieron unos criterios de conciencia interpretativa y descripción exhaustiva de los datos recogidos a través de entrevistas al profesorado, observaciones no participantes y análisis de documentos para asegurar la validez y triangulación de estos. Estos criterios fueron llevados a cabo por dos investigadores (CR, MR) durante el análisis de contenido de los resultados. En caso de discrepancia, estos investigadores consultaron a una tercera investigadora (AS) para mantener la fiabilidad y congruencia de los mismos (Noble y Smith, 2015). El sistema de códigos utilizados para la identificación de los diferentes datos obtenidos se recoge en la siguiente tabla (Tabla 4).

Tabla 4. Codificación para la identificación de los datos

\begin{tabular}{lll}
\hline \multicolumn{1}{c}{ Técnica } & \multicolumn{1}{c}{ Instrumento } & \multicolumn{1}{c}{ Informante } \\
\hline Entrevista semi-estructurada (E) & Guion de preguntas & Profesorado $(P)$ \\
Observación no participante (O) & Diario de Campo (DC) & \\
Análisis documental (AD) & Documentos (DO) & \\
\hline
\end{tabular}




\subsection{Aspectos éticos de la investigación}

Teniendo en cuenta las cuestiones éticas propias de una investigación inclusiva (Parrilla-Latas, 2017) y cumpliendo con los estándares establecidos en este tipo de investigaciones (AERA Council, 2011), se solicitó y se obtuvo el informe favorable por parte del Comité Deontológico de la Universidad Jaume I (CD/19/2020) antes de comenzar el proceso de investigación. Asimismo, y de forma previa al estudio, se facilitó el consentimiento informado a los participantes (Darretxe et al., 2020), adaptado a partir de Sales et al. (2019). Además de los principios éticos para la investigación en seres humanos acordados en la declaración de Helsinki y sus posteriores revisiones, en el proceso de recogida de datos se garantizó el anonimato, privacidad y confidencialidad de los mismos.

\section{Resultados}

Los resultados que se presentan dan respuesta al objetivo propuesto en esta investigación. De este modo, a partir del análisis de las unidades de significado, emergieron un conjunto de subtemas con similitudes conceptuales y, en último lugar, unos temas principales representados a partir de una red de relaciones semánticas (ver Figura 1). Asimismo, se seleccionaron un número de citas representativas para visualizar las ideas principales sobre los estilos y estrategias de enseñanza por parte de los docentes que participaron en el estudio.

Figura 1. Red de relaciones semánticas entre códigos

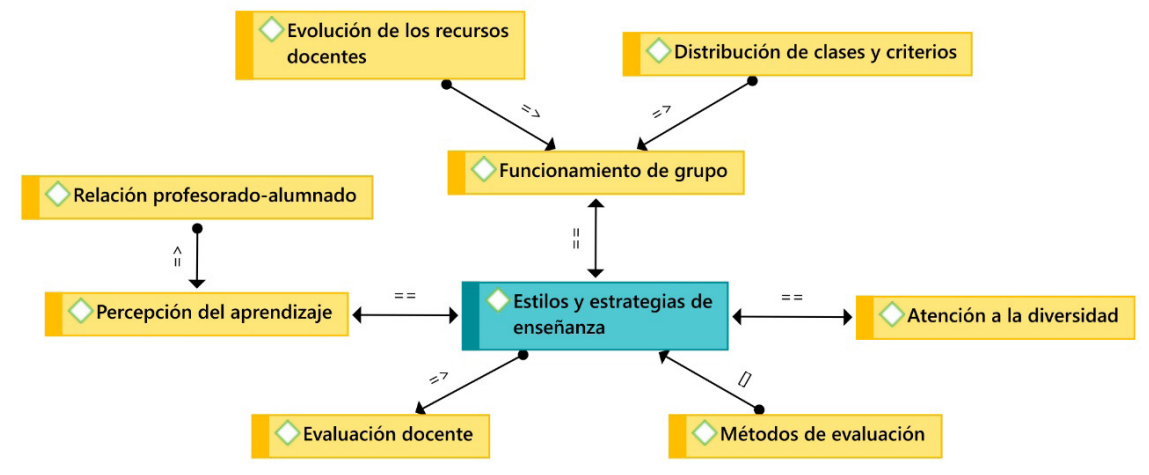

=>: es causa de; ==: está asociado con; [ ]: es parte de

\subsection{La puesta en práctica de las estrategias de enseñanza}

Este primer tema aglutina tanto la evolución en la organización que lleva a cabo el profesorado que imparte asignaturas en el ámbito de CCEE y Tecnología, como las diferentes dinámicas de clase y distribuciones utilizadas. De este modo, estos resultados revelan cómo las experiencias de los docentes influyen de forma determinante en el uso de recursos docentes para adaptarse a la variabilidad entre grupos de alumnos/as. 


\subsubsection{La necesidad del grupo como punto de partida}

Para la mayoría del profesorado, sus estrategias de enseñanza han ido cambiando no sólo a lo largo de su carrera profesional sino también en función de las necesidades que se iban encontrando en el alumnado (estilo reflexivo):

"Cuando empecé utilizaba fichas guiadas. Eso funcionaba, aparentemente, pero luego si les daba una ficha más abierta no eran capaces de hacerla porque estaban acostumbrados a un trabajo guiado. Así que intento presentar un proyecto abierto, para cada vez ir dándoles un poco más de autonomía. Yo me centro en que el alumno aprenda, que avancen un poquito" (E_P2)

Como indican la mayoría de los participantes, existe una necesidad de usar diferentes dinámicas en las clases, pero es necesario combinarlas (estilo reflexivo e individualizador). No todo sirve para todo, sino que hay que buscar aquellas que funcionan según el momento, la temática y el grupo de estudiantes a quienes van dirigidas:

"A mí me encanta explicar y sentir que los demás están aprendiendo, pero la verdad es que, dependiendo de en qué edades, necesitas utilizar otras estrategias para que ellos se motiven" (E_P4)

\subsubsection{Metodologías de enseñanza aplicadas en el aula}

En relación con las estrategias de enseñanza desarrolladas dentro del aula, los participantes describieron algunas que hacen referencia a estrategias de aprendizaje cooperativo, tanto de estructuras simples como complejas (estilo cooperativo):

"Como mi asignatura muchas veces es teórica, la lectura compartida me gusta mucho. Después de una lectura compartida, pregunto y la nota es igual para todos los del grupo, con lo cual todos tienen que estar atentos. Es una manera muy rápida de dar teoría. Y el Puzle deAronson también me gusta mucho"(E_P6)

Asimismo, mediante la observación y el seguimiento en las aulas se pudo contemplar el uso de estrategias integrales, además de estrategias de aprendizaje cooperativo (estilo individualizador, innovador y cooperativo):

"Los alumnos están distribuidos en grupos donde cada uno tiene diferentes tareas. Uno de los grupos está elaborando preguntas relacionadas con el tema para confeccionar las tarjetas de un juego final que se denominará "El cuerpo humano", otro grupo está elaborando las fichas y el tablero, y el último grupo está desarrollando las reglas del juego. La actividad a realizar por cada grupo rota en cada sesión" (O_DC-Sesión n7)

De la misma manera, durante las sesiones se desarrollaron estrategias de aprendizaje cooperativo que combinaban los grupos de investigación con un debate final (estilo reflexivo, indagador y cooperativo):

"Los alumnos están repartidos por grupos. Cada grupo debe buscar información relacionada con el nuevo virus, COVID-19, sobre cómo actúa, qué se conoce, cómo se puede prevenir el contagio, posible cura, entre otros aspectos" (O_DCSesión $n^{0} 10$ )

"Los alumnos están distribuidos en círculo con las anotaciones recogidas durante el proceso de investigación de la sesión anterior, debatiendo sobre los diferentes puntos planteados. Se escuchan entre ellos y discuten las diferentes fuentes de información, así como la información contrapuesta que presenta cada uno de los estudiantes" (O_DC-Sesión n¹2) 


\subsubsection{La distribución de clase como base en la enseñanza}

Como comentaban la mayoría de los participantes, no todos los grupos trabajan igual o las mismas estrategias de enseñanza funcionan en todos los grupos. Tal y como señalan estos docentes, los agrupamientos y las modalidades de trabajo adquieren un papel importante dentro de sus estrategias de enseñanza, dependiendo especialmente de la planificación de la sesión o sesiones (estilo cooperativo):

"Hay dinámicas grupales que son una sesión de clase y otras de dos. Luego, en otras asignaturas, estas dinámicas grupales se prolongan durante semanas, donde solemos hacer trabajos más largos y elaborados" (E_P1)

Así, la variabilidad en estas sesiones suele oscilar fundamentalmente entre una distribución grupal e individual (estilo individualizador):

"Hay actividades que son individuales y actividades grupales, dependiendo de la actividad. Por ejemplo, un mural, a lo mejor con dos es suficiente y si es una cosa más compleja, ya son grupos de tres o cuatro" (E_P3)

En este sentido y curiosamente, un criterio común para la formación de grupos que aparece en la mayoría de los participantes es la necesidad de formación de grupos heterogéneos, favoreciendo el aprendizaje vicario y aprendizaje entre iguales (criterio académico) (estilo reflexivo y cooperativo):

"Intento aplicar técnicas de juntar al que sabe con el que no. Intento que sean agrupaciones donde haya alguien que sea más hábil con alguien que no lo sea. Que no sea hábil a nivel de informática, no significa que no sea hábil en aportar ideas, con lo cual el tándem suele ser positivo" (E_P2)

"Los alumnos han realizado un mural de manera grupal sobre los animales vertebrados e invertebrados. En los murales hay diferentes animales en forma de "recortables" donde vas levantando las diferentes capas y puedes observar los órganos, el esqueleto, etc." (AD_DO)

La necesidad de formar estos grupos surge especialmente para fomentar las fortalezas del alumnado y poder trabajar de forma activa aquellos aspectos susceptibles de mejora de una manera más cercana (criterio personal) (estilo reflexivo y cooperativo):

"En todos los grupos hay tímidos. Debes colocar a gente muy activa, sabiendo que si pones a dos activos juntos van a explotar. En realidad, el grupo funciona si es diverso. Y te llevas sorpresas porque muchas veces esos que no funcionan solos y que sacan malas notas, resulta que son los que más animan [...]". Les haces sentirse mejor, elevas su autoestima y se portan bien" (E_P4)

\subsubsection{Evolución de los recursos docentes}

A pesar de que el uso de recursos docentes abarca un amplio abanico de posibilidades, muchos describieron cómo a lo largo de su experiencia profesional, la organización y uso de estos en sus clases también han ido cambiando. De esta forma, hoy no sólo se limitan a usar diapositivas (estilo innovador), sino también a acercar la realidad inmediata del alumnado a sus aulas desde diferentes perspectivas y aplicaciones (estilo innovador): 
"Si estoy trabajando un tema en concreto como el de posicionamiento web y conozco a gente del gremio, alguna vez hemos hecho una videoconferencia. Esa persona explica algunas cosas y ellos también le preguntan. Eso lo he hecho y ha funcionado súper bien" (E_P2)

"Si comparas un virus informático con un virus normal, lo entienden enseguida porque el virus informático es un parásito, y el virus también. Tienes que conectar tu asignatura con la realidad que ellos viven" (E_P5)

\subsection{Percepciones en la aplicación de estrategias de enseñanza}

Este segundo tema da respuesta a las propias percepciones de los docentes entrevistados sobre los resultados que han obtenido y obtienen en el uso de diferentes estrategias de enseñanza en el propio aprendizaje del alumnado, la conexión que se establece entre profesorado-alumnado y las experiencias para poder alcanzar una adaptación para la atención a la diversidad dentro del aula.

\subsubsection{Experiencias en base al método}

Con relación a las experiencias y percepciones que tiene el profesorado en base a las diferentes estrategias realizadas en las clases, la mayoría manifestó una experiencia positiva en el uso de estrategias de enseñanza que fuesen más allá de la transmisión directa de conocimiento (estilo individualizador):

"Cuando usaba algo diferente, vi que ellos lo agradecían y les ayudaba a organizarse. Me di cuenta de que tal y como lo estaba haciendo, se sentían un poco perdidos conmigo. Ahora puedo tener una opinión más clara sobre cada uno de mis alumnos, tengo más información sobre ellos y poco a poco intento que sean más autónomos" (E_P1)

De esta forma, se establece una relación enriquecedora entre profesoradoalumnado, ayudando a comprender que el proceso de enseñanza-aprendizaje también puede llegar a ser bidireccional (estilo reflexivo):

"A mí me ha enriquecido como persona porque recibes muchísimo más cariño. Creo que para aprender algo, tienes que creer que una persona te lo puede enseñar y, además, tiene que haber un hilo conductor emocional entre los dos, si no esa persona no aprende" (E_P4)

\subsubsection{La diversidad como experiencia docente}

En contraste, la experiencia en la atención a la diversidad entre los participantes no responde a una única estrategia de enseñanza (estilo cooperativo, reflexivo e individualizador):

"En cuanto a diversidad, alumnos que pueda tener que sean recién llegados, que no dominen el idioma, suelo ponerlos con personas que puedan ayudarles más y que ellos también puedan aportar" (E_P3)

Al igual que en la distribución de las clases, algunos de ellos/as optan por fomentar un aprendizaje entre iguales (estilo cooperativo), aunque también aparecen estrategias más pasivas para atender las necesidades del alumnado (estilo académico): 
"En vez de repartir fichas con adaptaciones curriculares, lo que hago es exigir menos capacidad de conceptos a aquellas personas que veo que tienen una capacidad memorística muy baja o que no son capaces de absorber los conceptos tan rápido como otros" (E_P5)

\subsection{La evaluación del conjunto}

Este último tema combina, por un lado, las experiencias sobre diferentes métodos de evaluación por parte del profesorado y, por otro lado, el uso de la evaluación docente como herramienta de mejora en las estrategias de enseñanza utilizadas en clase.

\subsubsection{La evaluación más allá del conocimiento}

La rúbrica aparece como una de las herramientas más utilizadas por el profesorado cómo método de evaluación (estilo individualizador):

"Desde hace tiempo utilizo la guía de evaluación y la rúbrica. Al principio, era una fan absoluta de la rúbrica, pero luego me he dado cuenta de que hay muchas veces que, si simplemente les das la guía, a ti no te encorseta tanto y a ellos también les das más margen a que te sorprendan" (E_P2)

"En los documentos recogidos sobre la manera de evaluar del profesorado de CCEE y Tecnología para su análisis, se pueden ver diferentes rúbricas de evaluación donde se incluyen los trabajos escritos, las exposiciones orales, el funcionamiento y comportamiento del grupo, la evaluación mediante rúbrica también de las sesiones de laboratorio, entre otras" (AD_DO)

No obstante, como indican muchos de estos docentes, la rúbrica sólo es una herramienta para un determinado momento, siendo necesario evaluar otros aspectos también esenciales en la formación del alumnado, utilizando para ello otros métodos (estilo individualizador y reflexivo):

"Utilizo rúbricas casi siempre. Depende de las cosas que esté haciendo porque luego hay un examen con una nota numérica que sería de conocimiento. Pero intento que todo lo demás cuente, porque si estamos haciendo una forma de enseñar que valore que tengan cierta adquisición de valores, no puedes obviarlo en la evaluación" (E_P6)

\subsubsection{La mejora a partir de la evaluación docente}

En lo relativo a la evaluación docente, se pudo observar que algunos profesores sí que la llevaban a cabo y les resultaba útil para realizar cambios o mejorar sus estrategias (estilo reflexivo y académico):

"Uno capta lo que opinan de uno. Sí que es cierto que a veces pregunto, "¿qué es lo que os ha resultado más difícil?" Esas cosas sí, pero no de una manera sistemática ni por escrito" (E_P5)

A pesar de que también existía profesorado que no hacía uso de la misma, los docentes que la utilizan parecen encontrar un buen recurso para poder cambiar y adaptar o, al menos detectar a tiempo, aquellas estrategias de enseñanza que no terminan de funcionar (estilo reflexivo e innovador): 
"Utilizo Google Forms y les pongo preguntas sobre “¿cuál es la unidad didáctica que has disfrutado más?, ¿cuál es la que has disfrutado menos?, ¿consideras que se podría mejorar?" Tengo una batería de preguntas que las voy refinando año tras año y las voy pasando. Les digo, "con lo que habéis vivido aquí, ¿cómo lo puedo mejorar para los alumnos del año que viene?" (E_P2)

\section{Discusión de los resultados y conclusiones}

\subsection{Aportaciones}

El objetivo de este estudio fue, por un lado, conocer qué estilos de enseñanza predominan entre el profesorado de Ciencias Experimentales y Tecnología (Biología y Geología, Física y Química, Tecnología e Informática) (CCEE) dentro de la Educación Secundaria Obligatoria (ESO) y Bachillerato, a través de las experiencias y perspectivas de los docentes, y cómo estos estilos influyen en las estrategias utilizadas en el aula. Por tanto, los resultados sugieren que no es posible enmarcar al profesorado en un determinado estilo de enseñanza, sino que se podría decir que los docentes tienen estilos de enseñanza dominantes o preferentes que van cambiando con el tiempo, la experiencia, la práctica reflexiva, así como adaptándose a las diferentes casuísticas que se pueden presentar dentro del aula (Delgado-Noguera, 1996; González-Peiteado, 2013). En la misma línea, Solbes et al. (2017) apuntan que el uso de estrategias de enseñanza activas, y, por tanto, el estilo de enseñanza predominante se ve influenciado por diferentes aspectos como la edad, las propias experiencias en las carreras profesionales de los diferentes docentes, el tipo de centro educativo o incluso las circunstancias contextuales de cada uno de ellos.

Además, los resultados arrojan datos interesantes sobre el uso de diferentes estilos de enseñanza entre el profesorado. De esta forma, se podría afirmar que el estilo predominante se enmarca en un estilo mixto de enseñanza, ya que una mayoría presenta estilos predominantemente activos o centrados en el alumnado, como el estilo individualizador, cooperativo o indagador. Estos resultados reflejan las mismas conclusiones que estudios ya existentes (Ballesta-Pagán et al., 2011), donde el profesorado con estilos de enseñanza preferentemente activos o centrados en el alumnado son percibidos por el alumnado como un perfil de buen profesor, con cualidades como respeto hacia el alumnado, cercanía y capacidad de escucha, entre otras. En este sentido, una posible explicación para este fenómeno podría ser el cambio en las propias estrategias de enseñanza, acercando la realidad del alumnado al temario, el uso de estrategias de aprendizaje cooperativo mediante grupos heterogéneos de estudiantes o el cambio de diferentes recursos docentes en base al funcionamiento del grupo (Waxman et al., 2015). En base a estos resultados, se puede apreciar que la mayoría de las estrategias de enseñanza utilizadas por los docentes que han participado en este estudio responden a enseñanzas de aprendizaje cooperativo. Sin embargo, el uso de otras estrategias igual de interesantes de acuerdo con la literatura consultada es bastante escaso, como el uso de estrategias integrales, estrategias de aprendizaje dialógico, estrategias de regulación y autorregulación del aprendizaje (Montealegre-García, 2019), quedando patente el largo camino aún por recorrer. Por otra parte, la utilidad de este cambio de enseñanza hacia unos estilos mixtos parece no 
limitarse exclusivamente a un cambio en aspectos didácticos, sino también como una necesidad educativa para poder atender a los diferentes ritmos y estilos de aprendizaje que estos docentes suelen encontrarse dentro del aula (Azorín-Abellán, 2018).

Por otro lado, es importante subrayar la percepción de los participantes en el uso de diferentes métodos de evaluación más allá del conocimiento. Si bien la mayoría de ellos utilizan diferentes herramientas dinámicas, como las rúbricas, en su proceso de evaluación (Buendía-García et al., 2016), también parecen incorporar otros aspectos igualmente importantes para la formación del alumnado, como la necesidad de conocer sus valores y actitudes que sin duda tendrán un papel fundamental en el día de mañana. De igual modo, este proceso de evaluación no parece limitarse a una evaluación unidireccional, sino que emerge como una oportunidad para mejorar las diferentes estrategias de enseñanza para algunos de ellos. Así, el uso de la evaluación docente aparece como un instrumento para mejorar el diseño de estrategias de enseñanza adaptadas a las necesidades de cada clase y, en definitiva, mejorar la calidad teniendo en cuenta ese carácter individual y personalizado que cada grupo puede necesitar (Alcón y Esteve-Mon, 2017). A pesar de ello, el profesorado parece quedarse en una fase inicial de la misma, sin llegar a la etapa de autoevaluación, donde la reflexión sobre la práctica docente constituye el pilar fundamental, con el fin de valorar la realización de mejoras o cambios sobre la misma (Martínez-Izaguirre et al., 2018; Vázquez-Bernal et al., 2007).

\subsection{Limitaciones y propuestas de mejora}

A pesar de todo lo comentado hasta el momento, hay una serie de limitaciones que se deben tener en cuenta a la hora de interpretar los resultados de este estudio. En primer lugar, la generalización de los datos es limitada, debido a que este tipo de estudios no garantizan la representatividad estadística y el estudio se llevó a cabo en un único centro. No obstante, a pesar de que participaron sólo 6 docentes, los resultados se fundamentan en la saturación de los datos durante su recogida, complementándose con el análisis documental y observaciones no participantes de las situaciones de aula. Asimismo, en base a la escasez en la literatura existente sobre las experiencias y percepciones de docentes en secundaria y bachillerato, la discusión de estos resultados también se vio limitada. Con todo ello, este trabajo no persigue bajo ninguna intención aportar una conclusión definitiva sobre el fenómeno de estudio. Al contrario, estos resultados ofrecen una oportunidad para abordar en mayor profundidad las experiencias y percepciones tanto del profesorado como del alumnado de secundaria y bachillerato de CCEE y Tecnología en materia de estrategias de enseñanza-aprendizaje para poder obtener una mayor comprensión del fenómeno de estudio. En definitiva, este estudio aporta nuevas pruebas sobre las perspectivas docentes en secundaria y bachillerato sobre los estilos de enseñanza y el uso de estrategias, teniendo en cuenta las diferentes casuísticas que cada docente y cada clase puede tener. 
Como propuestas de mejora, sería interesante promover formación continuada al profesorado sobre las diferentes metodologías activas existentes, como complemento y ampliación a las ya utilizadas por éstos en CCEE y Tecnología. De la misma manera, podría ser enriquecedor y proveer mejoras en la práctica docente, la formación e implementación de la autoevaluación por parte del profesorado de CCEE y Tecnología.

\subsection{Líneas futuras}

Como futuras líneas de investigación, podría ser interesante la implicación de más centros educativos con el objetivo de tener una visión más global sobre el fenómeno de estudio. Así mismo, sería interesante la realización de un trabajo más amplio donde se viesen más representadas otras áreas como las propias de las asignaturas de Tecnología, Informática y Física y Química. Finalmente, se considera relevante abordar la perspectiva desde el punto de vista del alumnado, con el fin de conocer la opinión y experiencias de todos los sujetos implicados en el proceso de enseñanza-aprendizaje.

A modo de conclusión cabe destacar la aproximación, cada vez mayor, del profesorado de CCEE y Tecnología hacia estilos y estrategias de enseñanza activos. Como se ha ido comentando a lo largo de los apartados de este trabajo, este cambio es muy positivo, ya que la influencia que tienen estos estilos y estrategias de enseñanza en la consecución de los logros académicos en los estudiantes y la mejora en las relaciones entre profesorado-alumnado es mayor, comparado con los estilos y estrategias más pasivas o académicas. Del mismo modo y en línea con los resultados de este trabajo, este cambio en el modo de enseñar conlleva la necesidad de acercar la realidad del alumnado a las aulas. Para ello, apoyado también en la literatura consultada, cabe señalar la necesidad de estrategias políticas, apoyo social y económico hacia una educación pública de calidad que permita a este profesorado adaptar sus estilos y estrategias de enseñanza, teniendo en cuenta la heterogeneidad de sus aulas, y con el objetivo final de ofrecer una educación inclusiva, equitativa y de calidad (Arnaiz-Sánchez et al., 2019). Por último, es importante subrayar el papel de la evaluación entendida como un proceso bidireccional en la labor docente. Por un lado, la evaluación docente surge como un instrumento fundamental para poder mejorar el diseño y calidad de los diferentes estilos y estrategias de enseñanza que podemos utilizar dentro del aula, pero también como un instrumento para ir más allá de la evaluación del conocimiento, poniendo los valores y actitudes que formarán a los ciudadanos del futuro en el centro. En definitiva, unos ciudadanos que tengan una alfabetización científica que les ayude a comprender y a mejorar el entorno científicotecnológico que les rodea (Cañal de León, 2012; Cañas et al., 2014; García-Barros et al., 2021; Garrido-Espeja y Simarro-Rodríguez, 2014; Verde-Romera et al., 2017).

\subsection{Implicaciones para el área}

A pesar de que existen diversos trabajos sobre estrategias de enseñanza en CCEE y Tecnología (Jiménez-Tenorio y Oliva-Martínez, 2016; Martin-Gámezetal., 2015), el tratamiento cualitativo a través de entrevistas a profesorado veterano aporta nuevas perspectivas sobre cómo ha evolucionado la docencia de este profesorado, cuáles han sido algunas de las barreras que han podido encontrar, cómo han ido adaptando sus estilos y estrategias a través de las necesidades identificadas, así como cuáles pueden ser futuras líneas para continuar con la formación permanente del profesorado. 


\section{Referencias}

AERA Council (2011). AERA Code of Ethics: American Educational Research Association. Educational Researcher, 40(3), 145-156. https://doi.org/10.3102/0013189X11410403

Aguilera, D., Lupiáñez, J., Vílchez-González, J.M. y Perales-Palacios, F.J. (2021). In Search of a Long-Awaited Consensus on Disciplinary Integration in STEM Education. Mathematics, 9(6), 597. https://doi.org/10.3390/math9060597

Aguilera-Morales, D., y Perales-Palacios, F.J. (2016). Metodología participativa en Ciencias Naturales: Implicación en el rendimiento y la actitud hacia la Ciencia del alumnado de Educación Primaria. ReiDoCrea, 5, 119-129.

Alcón, E., y Esteve-Mon, F.M. (2017). La importancia de la evaluación docente. Cuadernos de Pedagogía, 476, 1-3.

Alonso, C.M., Gallego, D.J. y Honey, P. (2012). Los estilos de aprendizaje: Procedimientos de diagnóstico y mejora. Ediciones Mensajero.

Álvarez-Álvarez, C., y San Fabián-Maroto, J.L. (2012). La elección del estudio de caso en investigación educativa. Gazeta de Antropología, 28(1). http://www.ugr.es/\%7Epwlac/G28_14Carmen_ Alvarez-JoseLuis_SanFabian.html

Arnaiz-Sánchez, P., De Haro-Rodríguez, R., Alcaraz-García, S., y Caballero-García, C.M. (2019). Escuchar las voces del alumnado para construir la inclusión y la equidad educativa: Diseño y validación de un cuestionario. Multidisciplinary Journal of Educational Research, 9(3), 258-292. https://doi.org/10.17583/remie.2019.4613

Azorín-Abellán, C.M. (2018). El método de aprendizaje cooperativo y su aplicación en las aulas. Perfiles educativos, 40(161), 181-195.

Ballesta-Pagán, F., Izquierdo-Rus, T. y Romero-Sánchez, B.E. (2011). Percepción del alumnado de Pedagogía ante el uso de metodologías activas. Educatio Siglo XXI, 29(2), 353-368.

Brailovsky, D. (2018). Lo nuevo y lo tradicional en educación: Una oposición engañosa. Revista Senderos Pedagógicos / ISSN: 2145-8243 / E-ISSN: 2590-8456, 9(9), 161-176.

Buendía-García, F., Benlloch-Dualde, J.V., Zahonero-Viana, I., y Cubel-Barea, A. (2016). Experiencias en la aplicación de tabletas en Secundaria. Education in the Knowledge Society (EKS), 17(4), 75-89.

Cañal de León, P. (2012). ¿Cómo evaluar la competencia científica? Revista Investigación en la Escuela, 78, 5-17.

Cañas, A., Martín-Díaz, M.J. y Nieda, J. (2014). Competencia en el conocimiento y la interacción con el mundo físico: La competencia científica. Alianza Editorial.

Contreras-Palma, S.A. (2009). Creencias curriculares y creencias de actuación curricular de los profesores de ciencia chilenos. Revista Electrónica de Enseñanza de las Ciencias, 8(2), 505-526.

Creswell, J. W. y Creswell, J.D. (2018). Research design: Qualitative, quantitative, and mixed methods approaches (Fifth Ediction). SAGE Publications.

Darretxe, L., Gezuraga, M., y Berasategi, N. (2020). La necesidad de avanzar hacia la investigación inclusiva. Márgenes Revista de Educación de la Universidad de Málaga, 1(1), 104-114. https://doi.org/10.24310/mgnmar.v1i1.7139

Delgado-Noguera, M.A. (1991). Los estilos de enseñanza en la educación física: Propuesta para una reforma de la enseñanza. Instituto de Ciencias de la Educación.

Delgado-Noguera, M.A. (1996). Aplicaciones a los estilos de enseñanza en Educación Primaria. En Estrategias metodológicas para el aprendizaje de los contenidos de Educación Física Escolar (pp. 73-86). Universidad de Granada.

Domingo-Roget, À. (2020). Profesorado reflexivo e investigador. Propuestas y experiencias formativas. Narcea.

Fernández-Navas, M. y Postigo-Fuentes, A.Y. (2020). La situación de la investigación cualitativa en Educación: ¿Guerra de paradigmas de nuevo? Márgenes Revista de Educación de la Universidad de Málaga, 1(1), 45-68. https://doi.org/10.24310/mgnmar.v1i1.7396 
Fuertes-Camacho, M.T. (2011). La observación de las prácticas educativas como elemento de evaluación y de mejora de la calidad en la formación inicial y continua del profesorado. Revista de Docencia Universitaria, 9(3), 237-258.

García-Barros, S., Martínez-Lossada, C., y Rivadulla-López, J. (2021). Actividades de textos escolares. Su contribución al desarrollo de la competencia científica. Enseñanza de las Ciencias. Revista de investigación y experiencias didácticas, 39(1), 219-238. https://doi.org/10.5565/ rev/ensciencias.3099

García-Cué, J.L., Sánchez-Quintanar, C., Velázquez-Jiménez, M., y Gutiérrez-Tapias, M. (2012). Estilos de aprendizaje y estrategias de aprendizaje: Un estudio en discentes de postgrado. Revista de estilos de aprendizaje, 5(10), 65-78.

García-Fraile, J.A., y Rojas-Aguilera, M. (2018). El portafolio de evidencias del alumno. Voces de la educación, 3(6), 177-190.

Garrido-Espeja, A., y Simarro-Rodríguez, C. (2014). El nou marc d'avaluació de la competència científica PISA 2015: Revisió i reflexions didàctiques. Ciències: Revista del professorat de ciències de Primària i Secundària, 28, 21-23. https://doi.org/10.5565/rev/ciencies.63

Generalitat Valenciana (2020). Institut Educació Secundària Juan Bautista Porcar. Institut Educació Secundària Juan Bautista Porcar. http://mestreacasa.gva.es/web/iesporcar/1

González-Peiteado, M. (2013). Los estilos de enseñanza y aprendizaje como soporte de la actividad docente. Revista de estilos de aprendizaje, 6(11), 51-70.

González-Peiteado, M., y Pino-Juste, M. (2016). Los estilos de enseñanza: Construyendo puentes para transitar las diferencias individuales del alumnado. Revista Complutense de Educación, 27(3), 1175-1191. https://doi.org/10.5209/rev_RCED.2016.v27.n3.47563

Ivic, S. (2016). Frequency of Applying Different Teaching Strategies and Social Teaching Methods in Primary Schools. Journal of Education and Practice, 7(33), 66-71.

Jiménez-Tenorio, N., y Oliva-Martínez, J.M. (2016). Aproximación al estudio de las estrategias didácticas en ciencias experimentales en formación inicial del profesorado de Educación Secundaria: Descripción de una experiencia. Revista Eureka sobre Enseñanza y Divulgación de las Ciencias, 13(1), 121-136.

Jorrín-Abellán, I.M., Fontana-Abad, M., y Rubi-Avi, B. (2021). Investigar en educación (1a Ed.). Síntesis.

Kallio, H., Pietilä, A.M., Johnson, M., y Kangasniemi, M. (2016). Systematic methodological review: Developing a framework for a qualitative semi-structured interview guide. Journal of Advanced Nursing, 72(12), 2954-2965. https://doi.org/10.1111/jan.13031

Martínez-Aznar, M.M., Martín-del Pozo, M.R., Rodrigo-Vega, M., Varela-Nieto, M.P., FernándezLozano, M.P., y Guerrero-Serón, A. (2002). Un estudio comparativo sobre el pensamiento profesional y la acción docente de los profesores de ciencias de educación secundaria: Parte II. Enseñanza de las Ciencias. Revista de investigación y experiencias didácticas, 20(2), 243-260.

Martínez-Bonafé, J. (1988). El estudio de casos en la investiación educativa. Investigación en la escuela, 6.

Martínez-Izaguirre, M., Yániz-Álvarez de Eulate, C., y Villardón-Gallego, L. (2018). Autoevaluación y reflexión docente para la mejora de la competencia profesional del profesorado en la sociedad del conocimiento. Revista de Educación a Distancia, 56, 31. http://dx.doi. org/10.6018/red/56/10

Martin-Gámez, C., Prieto-Ruz, T., y Jiménez-López, M.A. (2015). Tendencias del profesorado de ciencias en formación inicial sobre las estrategias metodológicas en la enseñanza de las ciencias. Estudio de un caso en Málaga. Enseñanza de las Ciencias. Revista de investigación y experiencias didácticas, 33(1), 167-184. https://doi.org/10.5565/rev/ensciencias.1500

Ministerio de Educación y Formación Profesional (2019). TALIS 2018. Estudio internacional de la enseñanza y del aprendizaje. Secretaría General Técnica. https://www.educacionyfp. gob.es/dam/jcr:047dfc41-acf6-444a-8e4e-4e5916343a88/talis2018-online-20190807.pdf

Monarca, H., y Rappoport, S. (2017). La experiencia de éxito académico como oportunidad para salir de situaciones de fracasos reiterados. Debates y Prácticas en Educación, 2, 23-35. 
Montealegre-García, C. (2019). Estrategias para la enseñanza y el aprendizaje de las ciencias (2ª Ed.). Universidad de Ibagué.

Montes de Oca-Recio, N., y Machado-Ramírez, E. (2011). Estrategias docentes y métodos de enseñanza-aprendizaje en la Educación Superior. Humanidades Médicas, 11(3), 475-488.

Morales, H., y Camacho-Verdugo, L.R. (2020). Filosofía de la Educación y pedagogía de la enseñanza en la formación del profesorado. Estudio de caso, percepción del estudiantado. Revista de la Universida de Costa Rica, 44(1 (enero-junio)), 19.

Navas, L., Soriano-Llorca, J.A., Holgado-Tello, F.P., y Jover-Mira, I. (2016). Las metas múltiples: Análisis predictivo del rendimiento académico en estudiantes chilenos. Educación XX1: Revista de la Facultad de Educación, 19(1), 267-285.

Noble, H., y Smith, J. (2015). Issues of validity and reliability in qualitative research. Evidence-Based Nursing, 18(2), 34-35. https://doi.org/10.1136/eb-2015-102054

Parra-Pineda, D.M., y Bustamante-Ramírez, M.V. (2003). Manual de estrategias de enseñanza / aprendizaje ( $1^{\mathrm{a}} \mathrm{Ed}$.). Ministerio de la Protección Social.

Parrilla-Latas, A. (2017). Ética para una investigación inclusiva. Revista de Educación Inclusiva, 3(1). https://revistaeducacioninclusiva.es/index.php/REl/article/view/218

Rapley, T. (2014). Los análisis de la conversación, del discurso y de documentos en investigación cualitativa. Morata.

Rekalde, I., Vizcarra, M.T., y Macazaga, A.M. (2014). La observación como estrategia de investigación para construir contextos de aprendizaje y fomentar procesos participativos. Educación XX1, 17(1), 201-220. https://doi.org/10.5944/educxx1.17.1.10711

Ruiz-Olabuénaga, J.I. (2012). Metodología de la investigación cualitativa (5ª Ed.). Universidad de Deusto.

Sales, A., Moliner, O., y Traver, J.A. (2019). Estrategias de investigación-acción participativa para la transformación. En B. Ballesteros (Ed.), Investigación social desde la práctica educativa (pp. 225-266). UNED.

Sandín-Esteban, M.P. (2003). Investigación cualitativa en educación. Fundamentos y tradiciones. MacGraw-Hill Interamericana de España.

Simons, H. (2009). Estudio de caso: Teoría y práctica. Morata.

Solbes, J., Fernández-Sánchez, J., Domínguez-Sales, M.C., Cantó, J.R., y Guisasola, J. (2017). Influencia de la formación y la investigación didáctica del profesorado de ciencias sobre su práctica docente. Enseñanza de las Ciencias. Revista de investigación y experiencias didácticas, 36(1), 25-44. https://doi.org/10.5565/rev/ensciencias.2355

Stake, R.E. (1998). Investigación con estudio de casos. Ediciones Morata.

Tong, A., Sainsbury, P., y Craig, J. (2007). Consolidated criteria for reporting qualitative research (COREQ): A 32-item checklist for interviews and focus groups. International Journal for Quality in Health Care, 19(6), 349-357. https://doi.org/10.1093/intqhc/mzm042

Vázquez-Bernal, B., Jiménez-Pérez, R., y Mellado-Jiménez, V. (2007). La reflexión en profesoras de ciencias experimentales de enseñanza secundaria: Estudio de casos. Enseñanza de las ciencias, 25(1), 73-90. https://doi.org/10.5565/rev/ensciencias.3761

Verde-Romera, A.M., Pablos-Miguel, M., y Caballero-Caballero, I. (2017). La competencia científica en los textos escolares. Un estudio LOE-LOMCE. Enseñanza de las ciencias: revista de investigación y experiencias didácticas, 0, 1129-1134.

Waxman, H., Weber, N., Franco-Fuenmayor, S., y Rollins, K. (2015). Research-Based Approaches for Identifying and Assessing Effective Teaching Practices. En Y. Li y J. Hammer, Teaching at Work. Advances in Teaching and Teacher Education. SensePublishers. https://doi. org/10.1007/978-94-6300-082-6_2 


\section{Anexo}

Modelo de entrevista

1. Variables sociodemográficas

Edad (años)

Será procesado en rangos de edad

Sexo

Hombre / Mujer

Nivel de estudios alcanzado

- Grado / Diplomatura / Licenciatura

- Máster

- Especificar:

- Doctorado

Especificar:

- Especificar:

Situación laboral actual

- Interino/a

- Fijo/a

- Sustitución

Experiencia laboral como docente (años / meses)

¿Ha recibido alguna formación continua (CEFIRE, cursos, etc.) recientemente? ¿Sobre qué temática?

2. Apertura

- Presentaciones:

- Comenzar con la presentación del entrevistador/a.

- Animar a presentarse, tanto en nombre como área en la que trabaja como docente.

- Explicar la duración de la sesión y la intención de la entrevista:

- Durará como máximo 30 minutos.

- Irá dirigida a obtener informaciones con los fines especificados en el consentimiento informado, para determinar las experiencias y perspectivas de docentes en el área de Ciencias Experimentales de Educación Secundaria Obligatoria y Bachillerato en el uso de estilos y estrategias de enseñanza.

- Establecer claras líneas de conducta:

- Las respuestas se dan en un clima de confianza y toda la información recogida será tratada con los estándares de privacidad y confidencialidad perceptivos de este tipo de investigaciones.

- Se alienta a expresar tanto perspectivas positivas como negativas, intentando fundamentar lo máximo posible sus respuestas. 


\section{Guion de preguntas}

1. Si tuviera que definirse como docente en una frase, ¿qué característica incluiría?

2. En esa frase, ¿qué valores y creencias considera que han influido e influyen en su modo de llevar a cabo la práctica docente?, es decir, ¿en qué creencias, valores o principios se sustentan tus prácticas didácticas?

3. Desde que comenzó a trabajar como docente, ¿cómo considera que han evolucionado sus estrategias de enseñanza?

4. ¿Cómo fue su primera experiencia como docente en el uso de estas estrategias? ¿cómo se ve ahora? ¿cómo cree que ha evolucionado?

5. De las estrategias utilizadas, ¿con cuál se siente más seguro/a o satisfecho/a? ¿o con cuál percibe que capta más al alumnado?

6. En cuanto a la gestión del espacio, tiempo, recursos (personales, materiales), agrupamientos, ¿qué criterio utiliza para hacer los grupos, parejas, etc.? ¿Y para organizar el aula u otros espacios? ¿Qué motivos suelen condicionar estos criterios?

7. Cuando lleva a cabo este tipo de dinámicas grupales, ¿cuánto tiempo suele llevarle? ¿varían mucho de unas dinámicas a otras? ¿qué resultados suelen tener?

8. Dentro de los diferentes tipos de tareas o actividades, ¿cuál usa frecuentemente? ¿por qué?

9. Ante la diversidad que hay hoy en día en las aulas, ¿cuáles son las estrategias que utiliza? ¿por qué?

10. Durante el curso académico, en sus clases, ¿participan las familias o algún otro agente comunitario que pueda servir como "recurso" en clase? Si participan, ¿cómo suelen llevarlo a cabo y cómo han sido las experiencias?

11. ¿Cuál es su percepción sobre la implicación y participación del alumno? ¿por qué piensa que muestran ese grado de implicación y participación?

12. En lo referente a la evaluación de los aprendizajes del alumnado, ¿qué instrumentos se utilizan?, ¿en qué momento se hace la evaluación?

13. Y en relación a la evaluación/retroalimentación de su tarea como docente por parte del alumnado, ¿utiliza algún instrumento? De ser así, ¿en qué momento se realiza?

14. ¿Cómo considera que es su relación con el alumnado en el día a día? ¿por qué?

15. ¿Querría añadir alguna información que considere relevante para el tema objeto de estudio que no se haya comentado?

\section{Preguntas de rescate}

1. ¿Qué estrategias utiliza para incrementar la autonomía del alumnado?

2. ¿Cómo percibe que es su rol como docente en el aula?

3. ¿Qué métodos utiliza para aumentar la participación e implicación del alumnado?

4. Resumen y conclusión

- Recapitular los puntos principales de la información recogida.

- $\quad$ Dar las gracias y ofrecer el poder añadir alguna información que consideren relevante para el tema objeto de estudio.

\section{Cómo citar en APA:}

Ropero-Padilla, C., Rodriguez-Arrastia, M. y Sanahuja, A. (2021). Aproximación a los estilos y estrategias de enseñanza del profesorado de la ESO y Bachillerato de ciencias experimentales y tecnología: un estudio de caso. Revista Iberoamericana de Educación, 87(1), 73-94. https://doi. org/10.35362/rie8714450 